\title{
First resolved observations of a highly asymmetric debris disc around HD 160305 with VLT/SPHERE ${ }^{\star, \star \star}$
}

\author{
Clément Perrot ${ }^{1,2,3}$, Philippe Thebault ${ }^{1}$, Anne-Marie Lagrange ${ }^{4}$, Anthony Boccaletti ${ }^{1}$, Arthur Vigan ${ }^{5}$,
} Silvano Desidera ${ }^{6}$, Jean-Charles Augereau ${ }^{4}$, Mickael Bonnefoy ${ }^{4}$, Élodie Choquet ${ }^{5}$, Quentin Kral ${ }^{1}$, Alan Loh ${ }^{1}$, Anne-Lise Maire ${ }^{15,8}$, François Ménard ${ }^{4}$, Sergio Messina ${ }^{7}$, Johan Olofsson ${ }^{8,2,3}$, Raffaele Gratton ${ }^{6}$, Beth Biller ${ }^{9,8}$, Wolfgang Brandner ${ }^{8}$, Esther Buenzli ${ }^{8,10}$, Gaël Chauvin ${ }^{4,11}$, Anthony Cheetham ${ }^{12}$, Sebastien Daemgen ${ }^{10}$, Philippe Delorme $^{4}$, Markus Feldt ${ }^{4}$, Eric Lagadec ${ }^{13}$, Maud Langlois ${ }^{14,5}$, Justine Lannier ${ }^{4}$, Dino Mesa ${ }^{16,6}$, David Mouillet ${ }^{4}$, Sébastien Peretti ${ }^{12}$, Pierre Janin-Potiron ${ }^{4}$, Graeme Salter $^{5}$, Elena Sissa ${ }^{6}$, Alain Roux ${ }^{4}$, Marc Llored ${ }^{5}$, Jean-Tristan Buey ${ }^{1}$, Alexei Pavlov ${ }^{8}$, Luc Weber ${ }^{12}$, and Cyril Petit ${ }^{17}$

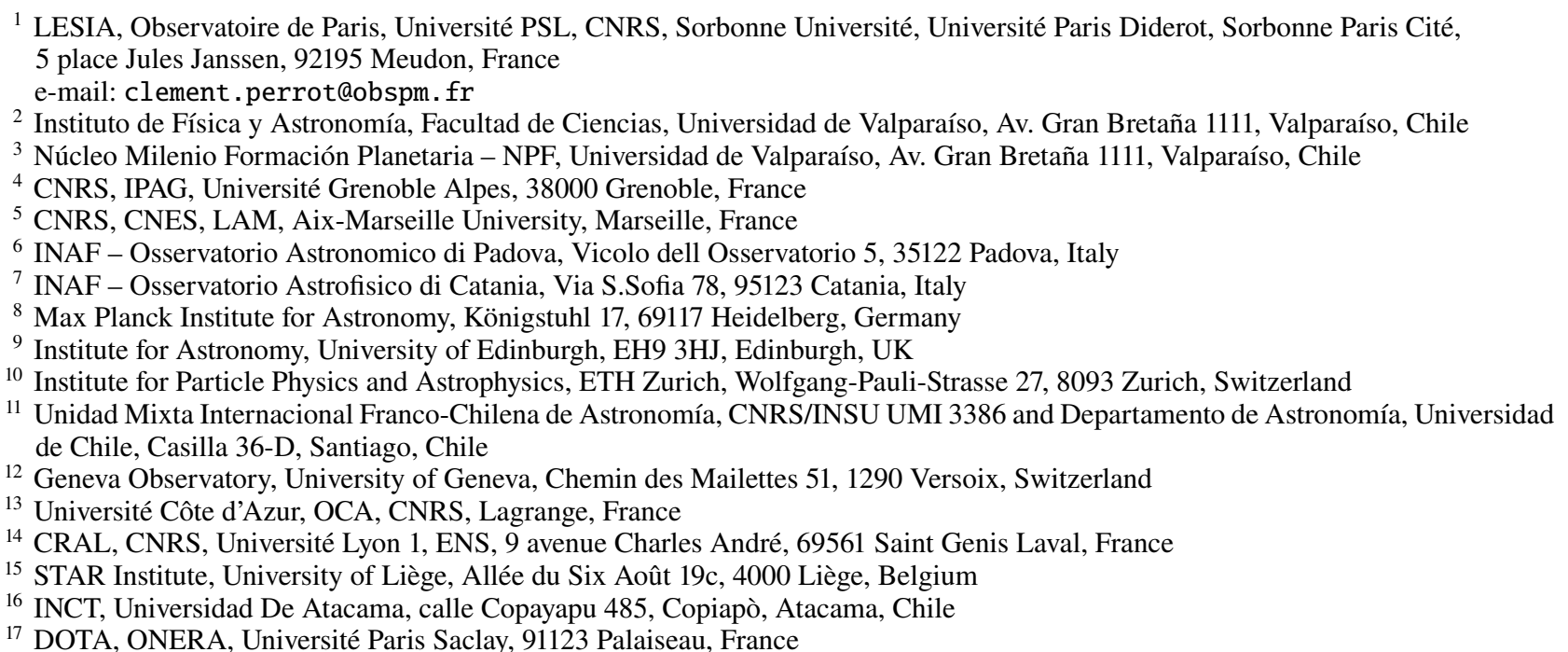

Received 19 November 2018 / Accepted 15 April 2019

\begin{abstract}
Context. Direct imaging of debris discs gives important information about their nature, their global morphology, and allows us to identify specific structures possibly in connection with the presence of gravitational perturbers. It is the most straightforward technique to observe planetary systems as a whole.

Aims. We present the first resolved images of the debris disc around the young F-type star HD 160305, detected in scattered light using the VLT/SPHERE instrument in the near infrared.

Methods. We used a post-processing method based on angular differential imaging and synthetic images of debris discs produced with a disc modelling code (GRaTer) to constrain the main characteristics of the disc around HD 160305. All of the point sources in the field of the IRDIS camera were analysed with an astrometric tool to determine whether they are bound objects or background stars. Results. We detect a very inclined $\left(\sim 82^{\circ}\right)$ ring-like debris disc located at a stellocentric distance of about 86 au (deprojected width $\sim 27 \mathrm{au}$ ). The disc displays a brightness asymmetry between the two sides of the major axis, as can be expected from scattering properties of dust grains. We derive an anisotropic scattering factor $g>0.5$. A second right-left asymmetry is also observed with respect to the minor axis. We measure a surface brightness ratio of $0.73 \pm 0.18$ between the bright and the faint sides. Because of the low signal-to-noise ratio $(\mathrm{S} / \mathrm{N})$ of the images we cannot easily discriminate between several possible explanations for this left-right asymmetry, such as perturbations by an unseen planet, the aftermath of the breakup of a massive planetesimal, or the pericenter glow effect due to an eccentric ring. Two epochs of observations allow us to reject the companionship hypothesis for the 15 point sources present in the field.
\end{abstract}

Key words. scattering - stars: individual: HD160305 - methods: observational - techniques: high angular resolution planet-disk interactions - techniques: image processing

\footnotetext{
* The reduced images (FITS files) are only available at the CDS via anonymous ftp to cdsarc.u-strasbg.fr (130.79.128.5) or via http://cdsarc.u-strasbg.fr/viz-bin/qcat?J/A+A/626/A95

$\star \star$ Based on observations made with ESO Telescopes at the Paranal Observatory under programs ID 95.C-0298 and 97.C-0865.
} 


\section{Introduction}

In the current model of planetary system formation, a circumstellar disc evolves from the protoplanetary disc phase (optically thick, dominated by gas; Williams \& Cieza 2011) to the debris disc phase (optically thin, with low or no gas content; Hughes et al. 2018). This inside-out process lasts typically a few million years during which the primordial gas dissipates, leaving only planets, if already formed, and planetesimal belts (Hernández et al. 2007). The latter trigger the production of a new generation of collisionally induced dust grains, potentially detectable in scattered light and thermal emission (see review of Hughes et al. 2018).

With the advent of dedicated instruments for high-contrast imaging (first the Hubble Space Telescope, see e.g. Schneider et al. 1999; Kalas et al. 2007; Choquet et al. 2016 and more recently the Spectro-Polarimetric High-contrast Exoplanet REsearch (SPHERE), Beuzit et al. 2019 and the Gemini Planet Imager (GPI), Macintosh et al. 2014), a variety of morphologies were discovered among debris discs. Several imaged discs present pronounced azimuthal asymmetries (HD 106906, Kalas et al. 2015; Lagrange et al. 2016, HD 61005, Hines et al. 2007; Olofsson et al. 2016 or GSC 07396-00759, Sissa et al. 2018). Other discs display several concentric belts, like HD 131835 (Feldt et al. 2017) or the hybrid disc (or potentially shielded disc of secondary origin, Kral et al. 2019) HD 141569 (Perrot et al. 2016). In some cases, very complex morphologies have been identified, like the very fast moving structures in the debris disc of AU Mic (Boccaletti et al. 2015, 2018). For most cases, the interaction between the disc and a potential massive companion has been invoked to explain these spatial structures (e.g. Lee \& Chiang 2016). However, apart from the particular case of $\beta$ Pictoris in which the planet $\mathrm{b}$ is known to have induced a disc feature (a warp, Mouillet et al. 1997; Augereau et al. 2001; Lagrange et al. 2010), the simultaneous detection of planet(s) and a disc is very uncommon in scattered light. Lee \& Chiang (2016) showed that a planet of a few Earth masses only is enough to create such asymmetries, yet such low-mass objects are beyond the reach of the current high-contrast imaging instruments. An additional example is the detected moving companion of Fomalhaut (Kalas et al. 2005, 2008), however, its planetary nature is still discussed (Neuhäuser et al. 2015; Poppenhaeger et al. 2017). For most imaged asymmetric discs, where no companion has been detected (yet), other explanations than the planet-sculpting scenario might also be possible. Such scenarios include the breakup of a massive planetesimal (Kral et al. 2015), or the interaction between dust and gas due for example to the photoelectric instability of the dust (Lyra \& Kuchner 2013; Richert et al. 2018).

HD 160305 (HIP 86598) is a F9V type star located at 65.51 \pm $0.23 \mathrm{pc}$ (previous value from HIPPARCOS: $72.46 \pm 4.5 \mathrm{pc}$, van Leeuwen 2007), with a proper motion of $-2.02 \pm 0.07 \mathrm{mas} \mathrm{yr}^{-1}$ in RA and $-65.86 \pm 0.06$ mas $\mathrm{yr}^{-1}$ in Dec (Gaia Collaboration 2018). The star was first proposed as a member of the $\beta$ Pictoris moving group (BPMG) by Kiss et al. (2011). This membership was later confirmed by Malo et al. (2013), but also contested by Song et al. (2012), who suggested that HD 160305 is a member of the Sco-Cen complex. However, we note that the star is quite far from this region in the sky, about $10^{\circ}$ apart from the closest group boundary as defined in Pecaut \& Mamajek (2016). The most recent analyses by Messina et al. (2017) and Lee \& Song (2018) further support the BPMG membership. Few other mild outliers are present within the bona-fide members, especially for stars with debris discs. Indirect age indicators like lithium and X-ray emission, although of limited sensitivity for an F9V star, are compatible with this membership as well as the isochrone fitting (Bell et al. 2015). Finally, using the recent kinematic data from Gaia Data Release 2 (DR2; Gaia Collaboration 2018) and the BANYAN $\Sigma$ tool $^{1}$ from Gagné et al. (2018), we obtain a probability of $99.4 \%$ that the star is a member of the BPMG. We thus adopt the age of the BPMG in the following: $23 \pm 3$ Myrs (Mamajek \& Bell 2014). Schneider (2013) reports a projected rotational velocity of the star $v \sin i=37 \mathrm{~km} \mathrm{~s}^{-1}$. Combined with the stellar rotation period $P=1.336 \pm 0.008$ days (Messina et al. 2017), we can derive a stellar rotation axis of $i_{\star}=58_{-10}^{\circ+18}$ (Appendix A). Kiraga (2012) also reported a small stellar variability at visible wavelengths, $\Delta I=0.041 \mathrm{mag}$ and $\Delta V=0.047 \mathrm{mag}$. A search in Gaia DR2 did not reveal any companions within 20 arcmin (that is $\sim 0.3 \mathrm{pc}$ at the distance of the star, Gaia Collaboration 2018). Gaia would reveal any companion with a $G$-band magnitude $<19.5$, which implies a mass larger than $\sim 0.15 M_{\odot}$ at the distance of HD 160305. While not fully complete, there is little room for stellar companions. Moreover, no gas was detected in this system, as reported by Moór et al. (2015).

The WISE ${ }^{2}$ survey (Pecaut \& Mamajek 2013) identified an infrared excess at $2.9 \sigma$ based on the $K s-W 4$ colour, confirmed by Moór et al. (2016) with Herschel observations at 70 and $160 \mu \mathrm{m}$. Using a single temperature spectral energy distribution (SED) fitting of the far-infrared excess, Moór et al. (2016) derived a first estimation of the disc's properties, with a radius at $58 \pm 13$ au, a disc temperature of $43 \pm 5 \mathrm{~K}$, and a fraction of dust luminosity with respect to the bolometric luminosity of the star of $1.2 \pm 0.3 \times 10^{-4}$. This system had never been imaged to date, neither in the submillimetric in thermal emission nor in scattered light. The SED-inferred disc radius corresponds to an angular size between $0.6^{\prime \prime}$ and $1^{\prime \prime}$, which is between the inner working angle and the field of view of SPHERE. Spatially resolving discs provides direct measurements of their radius and dust extent (e.g. ring versus extended disc) and allows us to break the degeneracies between the temperature and the distance to the star of the dust in SED fits.

In this paper we present the first resolved images of the faint disc around HD 160305, obtained in the near infrared with the SPHERE instrument. The observations are described in Sect. 2. The companionship analysis of the various point sources detected in our image is presented in Sect. 3. Section 4 presents the modelling of the disc. We then discuss in Sect. 4 the different scenarios that could explain the origin of the observed disc asymmetry.

\section{Observations}

HD 160305 was observed twice with SPHERE (Beuzit et al. 2019) as part of the guaranteed time observation (GTO) programme: SpHere INfrared survey for Exoplanets (SHINE; Chauvin et al. 2017).

On the first epoch (May 13, 2015) we used the standard IRDIFS mode combining simultaneously the Integral Field Spectrometer (IFS, Claudi et al. 2008) in the YJ mode $(0.95-$ $1.35 \mu \mathrm{m})$ and the Infra-Red Dual-beam Imager and Spectrograph (IRDIS, Dohlen et al. 2008) with the dual-band filters $H 2-H 3$ $\left(\lambda_{H 2}=1.593 \mu \mathrm{m}, \lambda_{H 3}=1.667 \mu \mathrm{m}, \delta \lambda=53 \mathrm{~nm}\right.$, Vigan et al. 2010). Sky conditions were good but we experienced some open loop interruptions of the adaptive optics control due to instabilities

\footnotetext{
1 Bayesian Analysis for Nearby Young AssociatioNs: http://www . exoplanetes . umontreal . ca/banyan/banyansigma.php

2 Wide-Field Infrared Survey Explorer.
} 
that required frame sorting during the data analysis. The disc was barely detected in this first data set as a very faint structure. To improve the detection of the disc, we used a hybrid configuration for the second epoch (May 23, 2016) where IRDIS was operated in classical imaging mode with the broad-band filter $B B \_H\left(\lambda_{\mathrm{c}}=1.625 \mu \mathrm{m}, \delta \lambda=290 \mathrm{~nm}\right.$, Langlois et al. 2014) in both spectral channels, and IFS still set up in the $Y J$ mode. Both observations were done with the 92.5 mas radius Apodized Lyot Coronagraph (APLC; Guerri et al. 2011). The fields of view of IRDIS and IFS are $11^{\prime \prime} \times 12.5^{\prime \prime}$ and $1.73^{\prime \prime} \times 1.73^{\prime \prime}$ respectively, with a pixel-scale of $12.242 \pm 0.062$ mas (first epoch) and 12.247 \pm 0.017 mas (second epoch) for IRDIS, and 7.46 \pm 0.02 mas for IFS (Maire et al. 2016). Using the NGC 6380 and NGC 3603 clusters as astrometric references, we measured the true north orientation of $1.712 \pm 0.063^{\circ}$ and $1.675 \pm 0.080^{\circ}$ for, respectively, the first and the second epoch. The setup and parameters of observations are summarized in Table B.1.

All SHINE observations follow a similar sequence: a first series of images of the star taken out of the coronagraphic mask and with a neutral density to avoid saturation. These images are used to calibrate the flux in the coronagraphic images (flux). Then, several coronagraphic data were acquired in waffle mode, which consists in applying a periodic modulation to the deformable mirror to create four crosswise spots around the star (Langlois et al. 2013). These spots allow us to determine precisely the position of the star behind the coronagraph (centring). Afterwards, a long series of deep coronagraphic exposures (science) was acquired, followed by a second series of coronagraphic images in waffle mode, a second unocculted star image to assess the flux variability during the sequence, and finally a few sky frames to subtract the bias, the dark, and the background from the deep coronagraphic exposures. All the remaining calibrations (darks, flats, wavelength calibration for IFS) are carried out in daytime. A GTO run comes with astrometric calibrator observations to determine the pixel scale and the true north orientation.

Both IRDIS and IFS data were reduced using the SPHERE data reduction and handling (DRH) pipeline (Pavlov et al. 2008), hosted at the SPHERE data centre (DC) in Grenoble ${ }^{3}$ (Delorme et al. 2017). The reduction applied to the data follows the standard procedure of SHINE targets including background subtraction, bad pixel and flat-field corrections, and centring of the coronagraphic frames using the "waffle" mode observation. The IRDIS acquisitions included a dithering pattern to help with bad pixel rejection. Additional custom routines were also used (frame centring and sorting), in particular for the IFS data reduction (Mesa et al. 2015). The IFS data were spectrally calibrated owing to internal calibration laser lines. The true-north (TN), the pixel-scale, and the distortion correction were determined for both epochs using the same method described by Maire et al. (2016). Sorting of coronagraphic frames was required for both epochs to remove open adaptive optics loops and poor-quality frames (poor adaptive optics corrections). A total of ten out of 64 frames, and 25 out of 144 frames, were rejected for the first and second epochs, respectively. Each data set is composed of a temporal and spectral cube of coronagraphic images, and of the unsaturated images of the star (point-spread function, PSF).

After the standard reduction, the data are post-processed with the SpeCal software (Galicher et al. 2018), hosted in the DC, which allows us to use a variety of post-processing methods based on angular differential imaging (ADI, Marois et al.

http://sphere.osug. fr/spip.php?rublique16\&lang=en
2006), spectral differential imaging (SDI, Smith 1987), or a combination of both. The reductions revealed the presence of several point sources and a resolved, though very faint, disc around the star. In this paper, we present the results obtained with post-processing based on the ADI method. We used the Template Locally Optimized Combination of Images (TLOCI, Marois et al. 2014) and the Karhunen-Loève Image Projection (KLIP, Soummer et al. 2012) algorithms, which provide, in this case, the best reductions for the point sources analysis and for the disc analysis, respectively (Figs. 1 and 4). For both epochs, the KLIP reductions are performed in a $2.5^{\prime \prime}$ radius field of view and a truncation of ten modes. The TLOCI reductions are performed for the entire images, using the same parameters as those used for the standard reduction of SHINE data, as defined in Galicher et al. (2018): Perc $=0.1$, the minimum throughput allowed for a point source; $\Delta r($ red $)=1.5 \lambda / D$, the width of the area where speckles are suppressed; $\Delta r(\mathrm{opt})=1.5 \lambda / D$, the width of the area where the TLOCI coefficients are calculated; $N_{\mathrm{A}}=20$, the size in PSF width-units of the area where speckles are suppressed.

We note that previous observations of the star were made with VLT/NaCo on the 1 and the 25 of June 2009 in the $K s$ band. Those observations were performed without coronagraph, saturating the central star to enhance the dynamical range of the data. Yet, the data quality was not sufficient to detect the disc.

\section{Point sources analysis}

The first-epoch observations revealed ten point sources in the field of view of IRDIS (Fig. 1, left), none of them are in the field of view of the IFS image. The second epoch confirms the detection of the previous ten point sources (Fig. 1, right: white circles) and adds five new detections (Fig. 1, right: black circles).

To determine the nature of these ten point sources (bound companions or stationary background stars), we measured their astrometry in the TLOCI images and computed their motion between the two epochs. The analysis of the proper motion plot (Fig. 2), which corresponds to the motion of each point source with respect to the proper motion of the star, allows us to conclude that none of the ten point sources are co-moving with the star, hence we can flag those as background stars.

Regarding the five new detections from 2016, we only have their $s$ magnitude and one epoch of astrometry, which is not enough to perform a proper motion analysis or a colourmagnitude diagram (CMD). The CMD compares the colour of a candidate to the colours of template dwarfs, computed from their published spectra and SPHERE filters transmissions, to determine the probability of a point source being a planet or a background star, when a proper motion analysis is not possible. The method is described in Appendix $\mathrm{C}$ of Bonnefoy et al. (2018). Candidates \#12, \#13, and \#14 are detected very close to the edge of the IRDIS image, with a projected separation up of $\sim 480$ au assuming they are co-moving with the star. Candidates \#11 and \#15 are very faint and have projected separations of $\sim 410$ and $\sim 150 \mathrm{au}$, assuming common parallax with the star. The status of these candidates is still undefined but based on their brightnesses and separations, their probabilities of being background stars are $100 \%, 72.3 \%, 100 \%, 98.8 \%$, and $89 \%$ for candidates \#11, \#12, \#13, \#14, and \#15, respectively, based on the Besançon Galaxy Model (Robin et al. 2003). The angular separation and the position angle of all companion candidates are reported in Table B.2.

Using the SpeCal pipeline (Galicher et al. 2018), we derived the contrast limits in $H 2, H 3$, and $B B \_H$ bands for IRDIS, and in $Y J$ for IFS, accounting for TLOCI/ADI photometric biases due to 

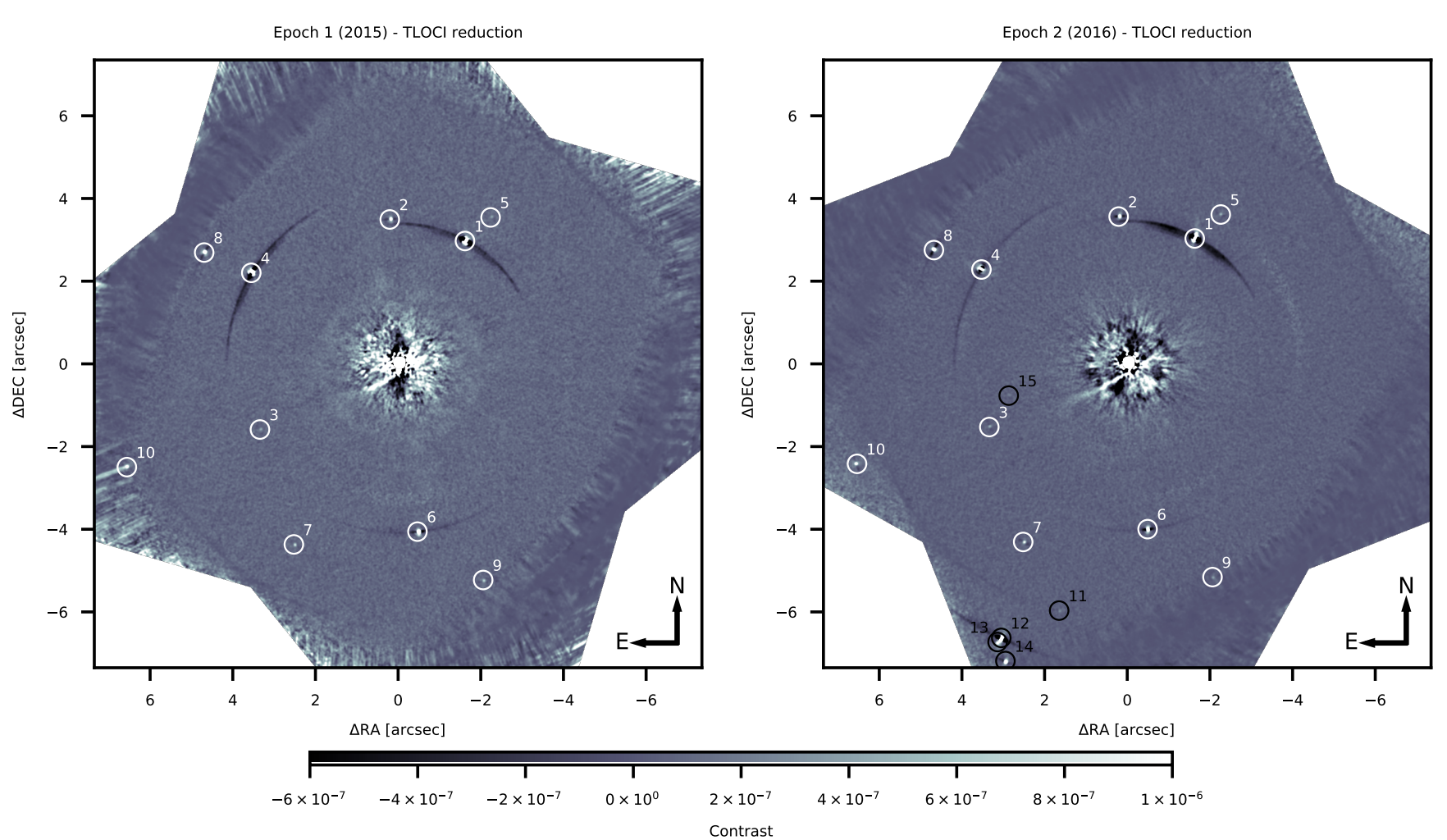

Fig. 1. IRDIS images of the first and second epochs, reduced with the TLOCI algorithm. North is up and east is left. Point sources are indicated with circles and numeric labels. Whites circles are used for point sources detected at both epochs and black circles for point sources detected at the second epoch only.

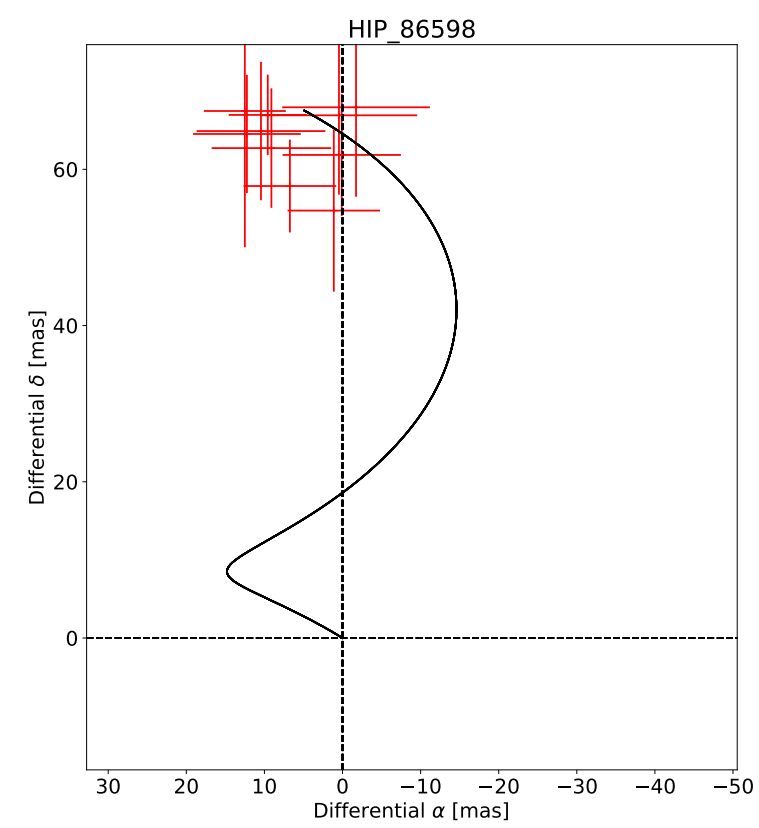

Fig. 2. Proper motion plot of point sources. Red crosses correspond to the RA/Dec motion of the point sources of the second epoch relative to the first epoch. The black line corresponds to the motion of a putative background star between the first and the second epochs. All motions of the point sources are compatible with the hypothesis of stationary background stars.

self-subtraction and for the coronagraph transmission. The contrast limits are presented in the top panel of Fig. 3. We converted the IRDIS contrast into detection limits expressed in Jupiter masses $\left(M_{\mathrm{J}}\right)$ using the BHAC-2015+COND evolutionary models (Baraffe et al. 2015) assuming an age of $23 \pm 3 \mathrm{Myr}$ (isochrones are interpolated for these given ages). These detection limits are presented in the bottom panel of Fig. 3. The detection limits in Jupiter masses were not computed for the IFS data, because the conversion from contrast to planet mass is dependent on the spectrum assumed for the planets that we aim to detect. The detection limits in $B B \_H(H 2)$ rule out the presence of companions more massive than $1 M_{\mathrm{J}}$ beyond angular separations of $2^{\prime \prime}\left(1.1^{\prime \prime}\right), 2 M_{\mathrm{J}}$ beyond angular separations of $1^{\prime \prime}\left(0.5^{\prime \prime}\right)$, and $4 M_{\mathrm{J}}$ beyond angular separations of $0.25^{\prime \prime}\left(0.25^{\prime \prime}\right)$. We note that due to the high inclination of the system (see Sect. 4), Jovian (resp. $2 M_{\mathrm{J}}$ and $4 M_{\mathrm{J}}$ ) companions at $80 \mathrm{au}$ (resp. $35 \mathrm{au}$ and $15 \mathrm{au}$ ) located angularly close to the star at the time of observations cannot be excluded.

\section{Debris disc analysis}

The first epoch of IRDIS data showed the weak detection of a disc seen at high inclination $\left(>80^{\circ}\right)$, with a position angle (PA) around $120^{\circ}$, at distances shorter than $1^{\prime \prime}$ from the star. Only the southeast side was detected in the first epoch. The detection of the disc was confirmed by the second epoch observations (Fig. 4, left) in which we found the same recurrent pattern on the southeast side, while the north-west side was revealed.

Figure 4 (right) shows the signal-to-noise ratio $(\mathrm{S} / \mathrm{N}) \mathrm{map}$, per element of resolution, of the disc for the KLIP reduction. To obtain this S/N-map, we binned the KLIP image in such a way that one pixel corresponds to a resolution element. Then we divided the binned image by a radial map of the standard deviation of the KLIP image measured in annuli (one resolution element width), which we use to estimate the noise in the 

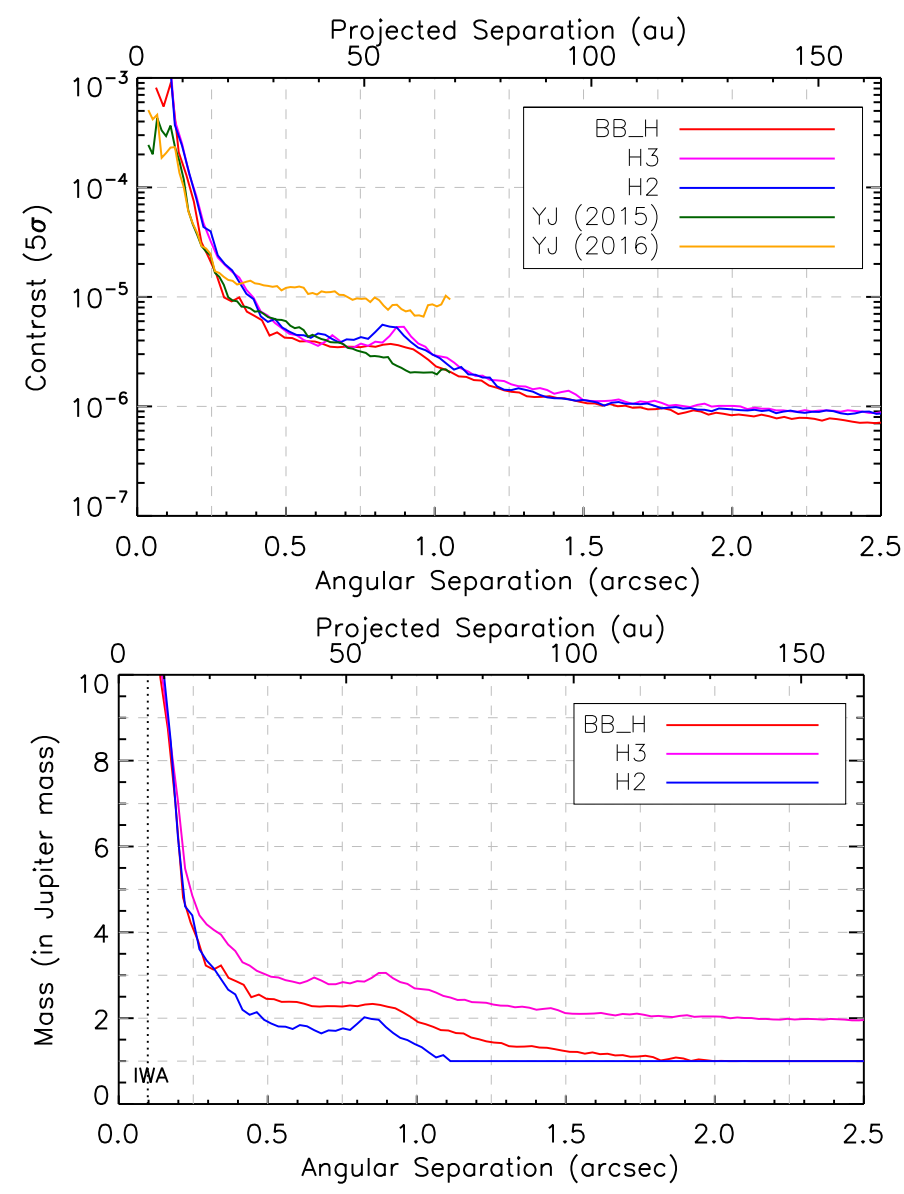

Fig. 3. Top: contrast limits at $5 \sigma$ for the IRDIS observation in $B B_{-} H$ (red), H3 (purple), H2 (blue) bands, and for the IFS observations in $Y J$ bands (green for 2015 and yellow for 2016). These contrast limits are obtained with the TLOCI reduction. Bottom: detection limits in planet masses obtained with the BHAC-2015+COND evolutionary models (Baraffe et al. 2015) for an age of $23 \pm 3 \mathrm{Myr}$, for the IRDIS $B B \_H$ (red), $H 2$ (purple), and $H 3$ (blue) bands. IWA correspond to the inner working angle of the coronagraph.

image (the disc was not masked). The disc is detected with a low S/N per resolution element, around 3-4 for the south-east part and 1-2 for the north-west part. These low $\mathrm{S} / \mathrm{N}$ can be explained by the nature of the noise (not Gaussian) at the position of the disc, which is dominated by large structures. These large structures are speckles that were not well suppressed by the data post-processing, which strongly affects the value of the noise at short separations and at the edge of the correction area of the adaptive optics $\left(\sim 1^{\prime \prime}\right)$. However, the south-east part of the disc is clearly different from the residual speckles pattern. We obtained an integrated $\mathrm{S} / \mathrm{N}$ of 16.8 for the south-east part. The integrated $\mathrm{S} / \mathrm{N}$ corresponds to the ratio between the integrated signal of the disc and the square root of the integrated square noise in the same area. This value represents the visual detectability of the disc and allows us to confirm the detection. For the north-west part, the detection is very marginal but it is the most compelling explanation according to the disc analysis made in Sects. 4.1, 4.2, and 4.3, assuming that the detected disc is a ring-like structure. We have chosen the assumption of a ringlike structure because the disc appears as a narrow curved feature with an offset from the central star. This observation is compatible with a ring-like structure or at least a belt, rather than an edge-on filled disc. However, other unusual configurations could be possible but cannot be confirmed or invalidated in this paper.

Assuming the ring-like structure of the disc, a brightness asymmetry in surface brightness is seen between the south-east part (the brightest) and the north-west part (barely detected). In the following, we will refer to this asymmetry as the twosided asymmetry. The disc is also asymmetrical on both sides of the major axis, the northern part being undetected. This "northsouth" asymmetry suggests that the southern part of the disc is the closest to the earth, assuming forward scattering dominates, while the northern part would be the backside of the disc. In the following, this second asymmetry will be referred to as the "frontward" asymmetry. Despite the self-subtraction of the disc caused by KLIP/ADI, we observe an expected decreasing brightness profile from small to large scattering angles. In several cases, the brightness profile can have a strong peak of scattering efficiency at very small scattering angles (Hedman \& Stark 2015; Milli et al. 2017).

\subsection{Disc model}

To derive the parameters of the disc, we used a model fitting method based on the GRaTer code (Augereau et al. 1999). Given the low $\mathrm{S} / \mathrm{N}$ of the disc, we could not directly measure the disc's scattering phase function. We used an analytical function to derive the disc's geometric parameters. The model assumes a radial surface density distribution described by Augereau et al. (1999), which decreases radially from $R_{0}$, the disc radius (in au), inwards and outwards as a power law with slopes $\alpha_{\text {in }}$ and $\alpha_{\text {out }}$. We used the grain scattering phase function defined by Henyey \& Greenstein (1941), parametrized by the coefficient of anisotropy of scattering $g$ (defined between -1 and $1,-1$ meaning backward scattering and 1 meaning forward scattering). We note $i$ the inclination of the disc (in degrees), PA the position angle (in degrees), and $h$ the aspect ratio between the height of the disc and its radius $R_{0}$. The simulated disc has no eccentricity, but it is possible to add two orthogonal offsets to simulate the effect of a small eccentricity of the disc (at first order).

Our method to characterize the disc is similar to the one used in Perrot et al. (2016). We generate a grid of models with different parameter values (Table 1), for a total of 69120 models. Several steps of the optimization process are illustrated in Fig. 5, comparing the best model to the KLIP-processed IRDIS data from the second epoch. Each model is convolved with the stellar PSF for comparison with the data (Fig. 5a). The models are then projected onto the eigenvectors of the coronagraphic images to apply the same biases (self-subtraction, Milli et al. 2012) as those affecting the data (Fig. 5c), similar to the forward modelling approach used by Choquet et al. (2016). To avoid oversampling in the optimization process, we convolved the data and the model with a Gaussian of three pixels, corresponding to one resolution element.

Then, each model is subtracted from the data (Fig. 5d) with an intensity scaling factor to match the surface brightness of the model to the surface brightness of the disc (pixel to pixel). This intensity scaling factor is computed, using the Amoeba function of IDL, on the brightest part of the disc only (the south-east part), using a mask (Fig. 5e) applied to the data (Fig. 5f). Finally, for each model we estimate the goodness of fit $\left(\chi^{2}\right)$ to determine which models are the closest to the observations. The minimization is performed in the eastern part of the disc, with a specific mask (Fig. 5g) applied to the residuals between the model and 

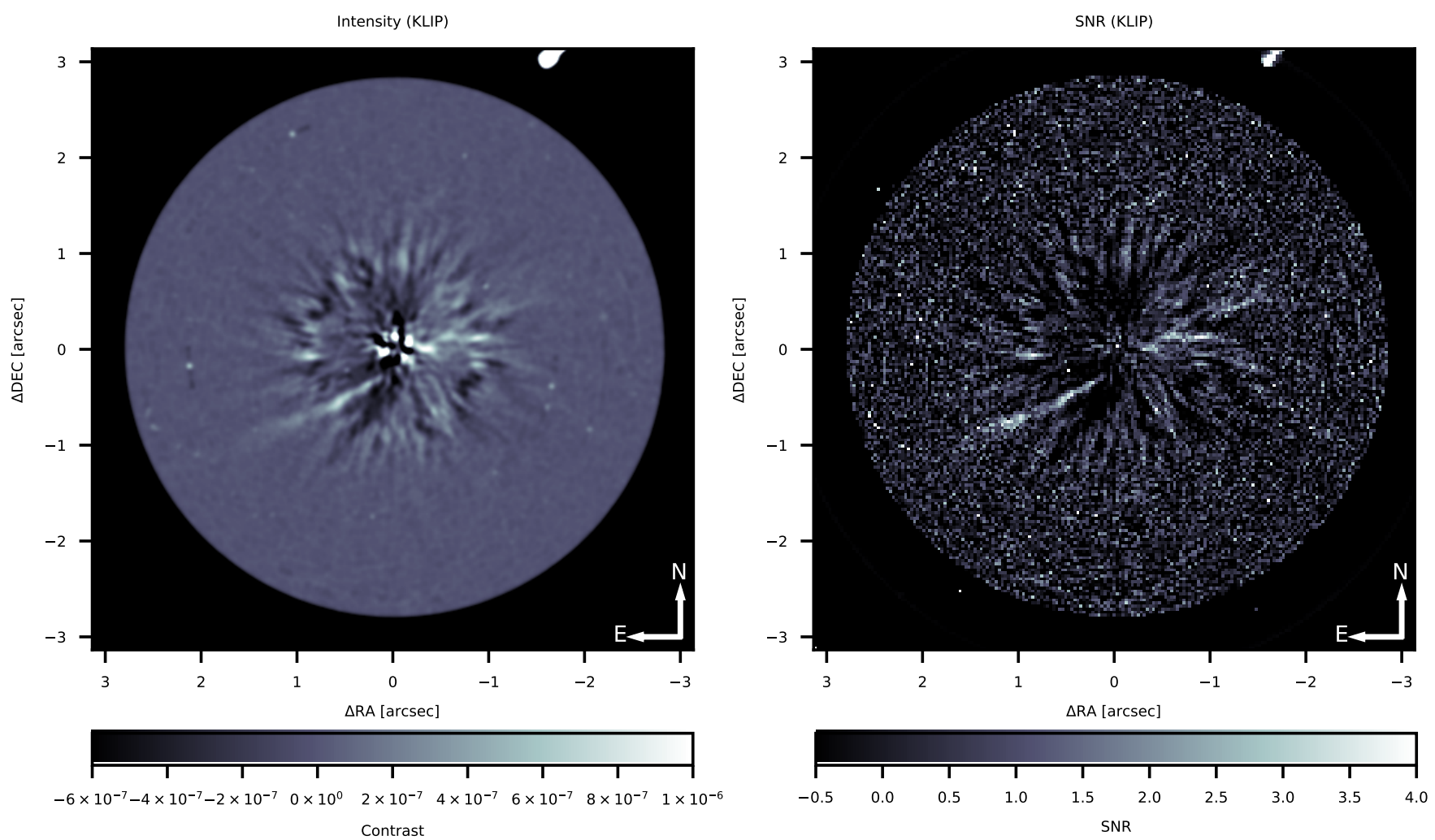

Fig. 4. Left: zoom into the disc in the KLIP reduction of the May 2016 data. North is up, east is to the left. Right: signal-to-noise map derived from the KLIP reduction (same orientation).

Table 1. Parameters used to generate the grid of models.

\begin{tabular}{ccc}
\hline \hline Parameters & Values $^{(1)}$ & Best model $^{(2)}$ \\
\hline$R_{0}(\mathrm{au})$ & {$[81 ; 86 ; 90 ; 95 ; 99 ;$} & 86 \\
& $104 ; 109 ; 113 ; 118]$ & \\
$\alpha_{\text {in }}$ & {$[2 ; 5 ; 10 ; 20]$} & 10 \\
$\alpha_{\text {out }}$ & {$[-2 ;-5 ;-10 ;-20]$} & -5 \\
$i\left(^{\circ}\right)$ & {$[81 ; 82 ; 83 ; 84 ; 85]$} & 82 \\
PA $\left(^{\circ}\right)$ & {$[120.5 ; 121 ; 121.5 ; 122 ;$} & 122.5 \\
& $122.5 ; 123 ; 123.5 ; 124]$ & \\
$g$ & {$[0.25 ; 0.5 ; 0.75]$} & 0.5 \\
$h$ & {$[0.005 ; 0.01 ; 0.025 ; 0.05]$} & 0.005 \\
\hline
\end{tabular}

Notes. ${ }^{(1)}$ Values of parameters to generate the grid of models. ${ }^{(2)}$ Values of the best model after a $\chi^{2}$ minimization.

the data (Fig. 5h). We used the eastern part of the disc instead of the full disc because the south-east to north-west surface brightness asymmetry of the disc cannot be reproduced with our symmetric debris disc model and because the northern part of the disc has a very low $\mathrm{S} / \mathrm{N}$.

\subsection{Best model}

The parameter values corresponding to the best model are given in Table 1. The bright south-east part of the disc is well fitted by the best model. The north-west part of the disc is over-fitted by the model (negative pattern in Fig. 5d), which confirms the observed two-sided asymmetry in intensity.

The frontward asymmetry, probably caused by forward scattering, is well reproduced both in the northern and in the southern part of the disc. For this model the best value found for $g$ is 0.5 , but we note that the sampling of $g$ values in our grid is coarse.

To estimate the uncertainty on these values, we plot in Fig. 6 the histogram of the $1 \%$ best models for each parameter (corresponding to 691 models). It is important to note that some of these $1 \%$ best models are visually not very consistent with the disc image, but still have low $\chi^{2}$ values. This issue is mainly due to the low $\mathrm{S} / \mathrm{N}$ of the disc and to the poorly fitted two-sided asymmetry. Therefore, these histograms provide a trend for the goodness of fit of each parameter value. In addition, we plot (as a red dashed line) the Gaussian fit of each histogram, except for the $g$ values due to the coarse sampling. Once again, because of the low $\mathrm{S} / \mathrm{N}$ in many regions of the image, these fits should be taken with caution, especially for $\alpha_{\text {in }}, \alpha_{\text {out }}$, and $h$. The mean values and the standard deviation of the Gaussian fits are reported in Table 2.

The Gaussian fit for $\alpha_{\text {out }}$ favours values around -5 to -10 . The situation is less clear for $\alpha_{\text {in }}$, for which the Gaussian fit has a large width, with a mean around 10 . These results are not surprising because the S/N quickly becomes very poor beyond the main ring. However, these measurements are sufficient to confirm the ring-like nature of the disc, since the slope of the surface density gives a full width at half maximum (FWHM) around 27 au (Fig. 7). The preferred values for the $g$ parameter are systematically higher than 0.5 but rarely higher than 0.75 . For the radius of the disc, $R_{0}$, the histogram is very well fitted by a Gaussian with a mean at $\sim 90$ au and a $\sigma_{R_{0}} \sim 10 \mathrm{au}$. For the $h$ ratio, we can only estimate an upper limit of 0.025 , but cannot discriminate between the thinner configurations 0.005 or 0.01 , mainly because the $h$ ratio is constrained by the limited beam resolution. The value 0.025 is also present in $25 \%$ of cases. This $h<0.025$ constraint is still compatible with the minimum "natural" scale height, $h \sim 0.04 \pm 0.02$, for debris discs in which 
(a) Best Model

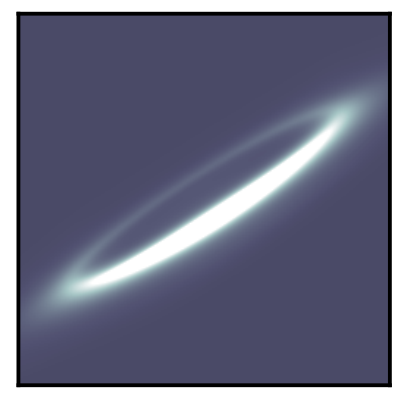

(c) Best Model (adi effect)

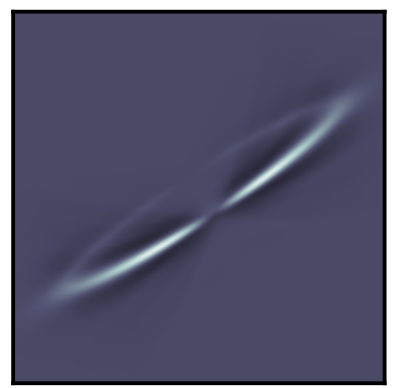

(e) Mask for the flux fitting

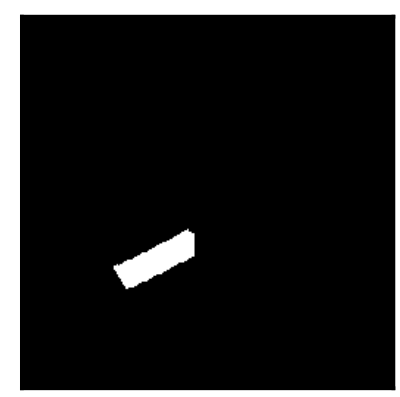

(g) Mask for the optimisation

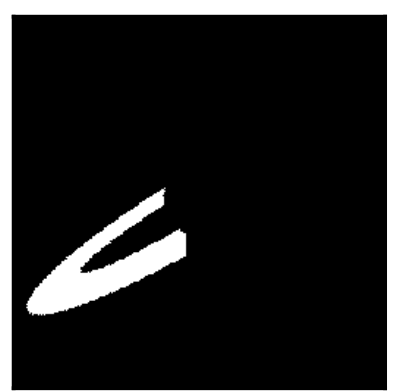

(b) Data

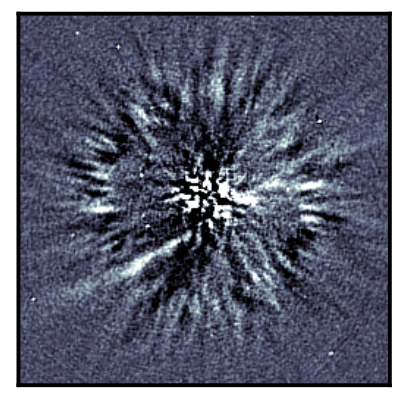

(d) Data - Best model

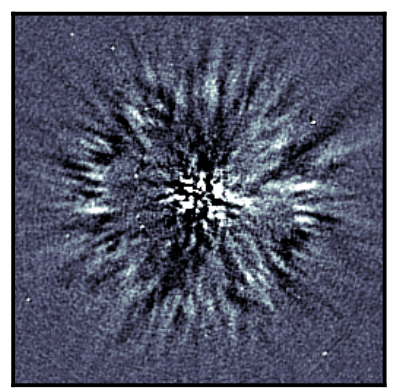

(f) Flux fitting zone

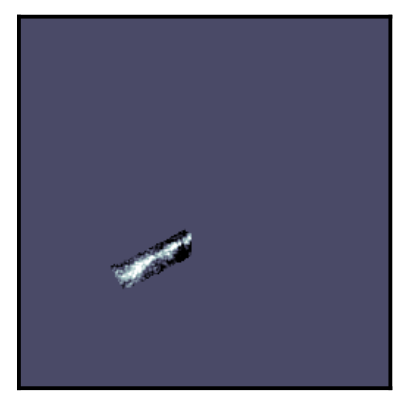

(h) Optimisation zone

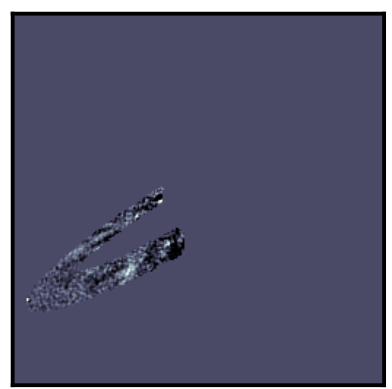

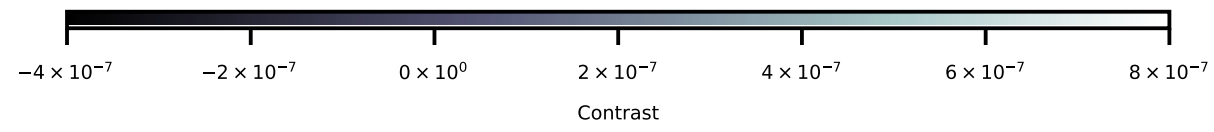

Fig. 5. Masks, data, and models used to determine the best model of the disc. $a$ : best model of the disc. $b$ : KLIP-reduced data. $c$ : best model of the disc after KLIP/ADI reduction. $d$ : data subtracted by the best model, after KLIP/ADI reduction. $e$ : mask used to select the part of the disc where the intensity scaling is done. $f$ : mask applied to the data for the intensity scaling of the disc model. $g$ : mask used to select the part of the disc where the $\chi^{2}$ minimization is done. $h$ : mask applied to the data, subtracted by the best model, for $\chi^{2}$ minimisation. Masks e and g are based on approximate ellipse parameters, close to the disc parameters.

small dust grain dynamics is controlled by radiation pressure (Thébault 2009). The inclination $i$, which is $82^{\circ}$ for the best model of the grid, is estimated to $82.4 \pm 0.8^{\circ}$ with the Gaussian fit. Finally, the position angle PA is constrained with a mean at $122.4^{\circ}$ and a dispersion $\sigma_{\mathrm{PA}}=2^{\circ}$. This result is still in agreement with the best model of the grid $\left(122.5^{\circ}\right)$ and should be better constrained with new deeper observations. Using the values obtained for $\alpha_{\text {in }}$ and $\alpha_{\text {out }}$ with the best model, we determined the radial profile of the disc (Fig. 7). If seen face-on, the disc would have a FWHM of 27 au (red line). With an inclination of $82^{\circ}$ the front of the disc (along the semi-minor axis) would have a FWHM of 6.4 au (blue line).

\subsection{South-east to north-west surface brightness ratio}

We measure the surface brightness ratio between the south-east and north-west parts $\left(\frac{\Phi_{E}}{\Phi_{W}}\right)$ to quantify the two-sided asymmetry. To do so, we used the best model derived from the previous analysis, normalized to different surface brightness levels, then subtracted from the raw data before the post-processing (negative injection of a fake disc in raw data). We used surface brightness factors ranging from 0 to $5 \times 10^{-6}$ with steps of $10^{-8}$ in terms of contrast. With this analysis, we can determine the surface brightness of the south-east and of the north-west sides independently and compute the surface brightness ratio between them.

More precisely, for each tested surface brightness value we inject a negative model in each raw frame at the same PA as the real disc (taking into account the field rotation), then we apply the KLIP post-processing algorithm with a truncation at ten modes. Therefore, the disc is more or less removed in the final obtained image, depending on the value of the injected surface brightness. To determine the optimal surface brightness factor, we minimize the mean squared error $(\Sigma)$ in the residuals inside two specific masks, one for each side of the disc.

In Fig. 8, we plot this criterion versus the surface brightness factor for both sides. The minimum of each plot gives the optimal surface brightness factor for the south-east and for the north-west side. The surface brightness ratio is then the ratio between these two coefficients. We find a value of $\frac{\Phi_{E}}{\Phi_{W}}=0.73 \pm$ 0.18 . The uncertainty is given by a combination of the error $\left(3.5 \times 10^{-7}\right)$ due to the noise inside the correction area, which extends from 250 mas to $1^{\prime \prime}$ and corresponds to the zone where the disc is located, and the systematic error $\left(10^{-8}\right)$ for the method, which corresponds to the step of the evaluated surface brightness factors. Of course the dominant contribution is due to the error caused by the residual speckle subtraction in the correction area.

With this analysis, we are also able to determine the flux ratio, in scattered light, between the star and the disc $\frac{\Phi_{\text {disc }}}{\Phi_{\star}}$. To do so, we integrated the intensity of an off-axis image of the star (which is not saturated) and the surface brightness of the best model, which is adjusted in intensity to correspond to the real disc. We find $\frac{\Phi_{\text {disc }}}{\Phi_{\star}}=1.4 \pm 0.22 \times 10^{-4}$. We note that this value is relatively close to the one derived for the thermal emission fractional luminosity by Moór et al. (2016), $1.2 \pm 0.4 \times 10^{-4}$. 

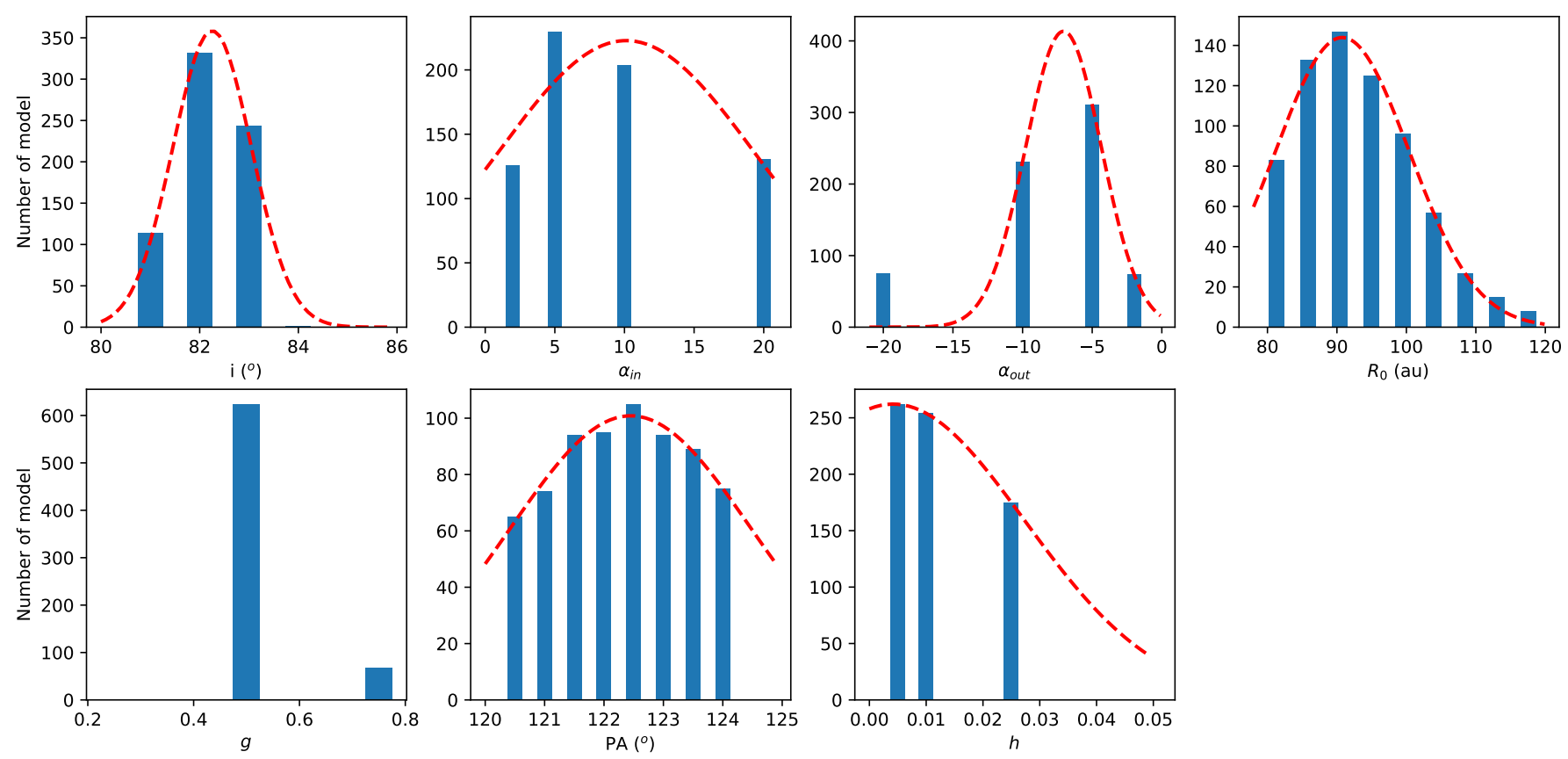

Fig. 6. Histogram of the $1 \%$ best model for each parameter tested. The bars correspond to the values used for the model grid and presented in the Table 1.

Table 2. Parameter values obtained with the $1 \%$ best models.

\begin{tabular}{cc}
\hline \hline Parameters & Values \\
\hline$R_{0}(\mathrm{au})$ & $90.8 \pm 9.6$ \\
$\alpha_{\text {out }}$ & $-7.1 \pm 2.7$ \\
$\alpha_{\text {in }}$ & $10.1 \pm 9.3$ \\
$\Delta R(\mathrm{au})$ & 27 \\
$i\left(^{\circ}\right)$ & $82.3 \pm 0.8$ \\
$\mathrm{PA}\left(^{\circ}\right)$ & $122.4 \pm 2.0$ \\
$g$ & $\sim 0.5$ \\
$h$ & $<0.025$ \\
\hline
\end{tabular}

Notes. The parameter $\Delta R$ corresponds to the FWHM of the ring, derived from the $\alpha_{\text {in }}$ and $\alpha_{\text {out }}$ parameters of the best model. The $R_{0}, \alpha_{\text {in }}, \alpha_{\text {out }}, i$, and PA values and errors are determined by fitting a Gaussian to the histogram (red dashed line). Due to the low number of points for $\alpha_{\text {out }}$ and $\alpha_{\text {in }}$, these values should be taken with caution. Moreover, $g$ and $h$ do not have enough points to perform a Gaussian fitting.

\section{Discussion}

The disc presents two kinds of asymmetries: the frontward asymmetry, between north and south, and the two-sided asymmetry, between south-east and north-west. The first one can easily be explained by a forward scattering effect of the dust, making the front side of the disc brighter than its back side (Hughes et al. 2018). In this case, the effect of scattering can be modelled, in the same way as in Sect. 4, where we found a value of the g-factor compatible with the forward scattering effect. As for the southwest to north-east asymmetry, corresponding to an azimuthal asymmetry in the debris ring, there are several possible explanations, which can be divided into two categories: scenarios assuming there is physically more dust in the brighter regions, and those assuming that these regions are bright because they are closer to the star.

If the south-west side corresponds to a physical local overdensity, a first explanation is the perturbations of a massive

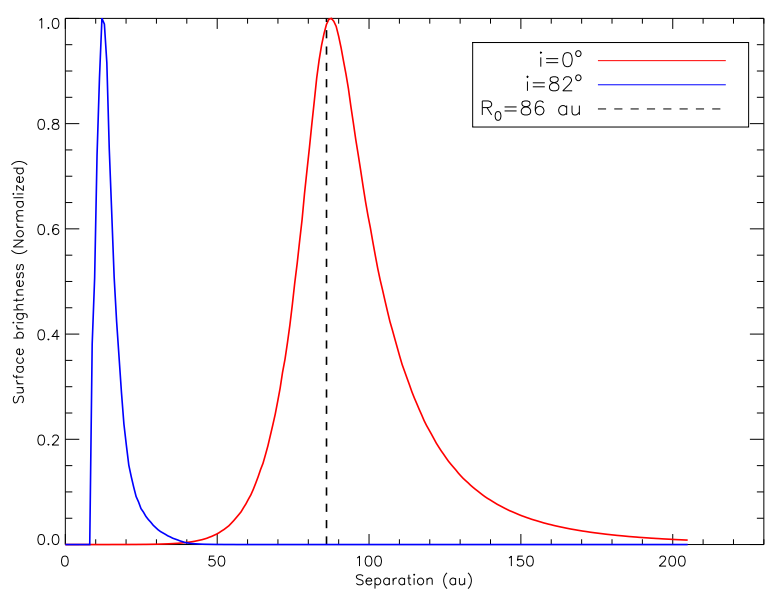

Fig. 7. Normalized radial profiles for the best model disc. Red line: deprojected disc (face-on). Blue line: viewing angle of $82^{\circ}$. The FWHM for the deprojected and inclined configurations are 27 and $6.4 \mathrm{au}$, respectively. The black dashed line corresponds to the position of $R_{0}$.

planet, which can create azimuthal inhomogeneities at the location of mean motion resonances (e.g. Kuchner \& Holman 2003; Reche et al. 2008; Thebault et al. 2012). The presence of such a giant planet sculpting the main ring is not detected in our data after subtraction of the best disc model (Fig. 5d). If there is a planet inside the disc, its mass is below our detection limits $\left(1-2 M_{\mathrm{J}}\right)$ or the planet is hidden by the residuals close to the star. However, super-earth mass planets, which are below our detection limits (see Sect. 3), can also create such disc asymmetries (Lee \& Chiang 2016), and this explanation for the asymmetry thus cannot be ruled out. The planetary hypothesis could be compounded by the fact that there seems to be a sharp drop in surface brightness beyond the main ring, corresponding to a surface density decreasing with a slope $-10 \lesssim \alpha_{\text {out }} \lesssim-5$ that is much steeper than the canonical -1.5 slope expected for a small grain halo beyond an unperturbed ring (Strubbe \& Chiang 2006; 


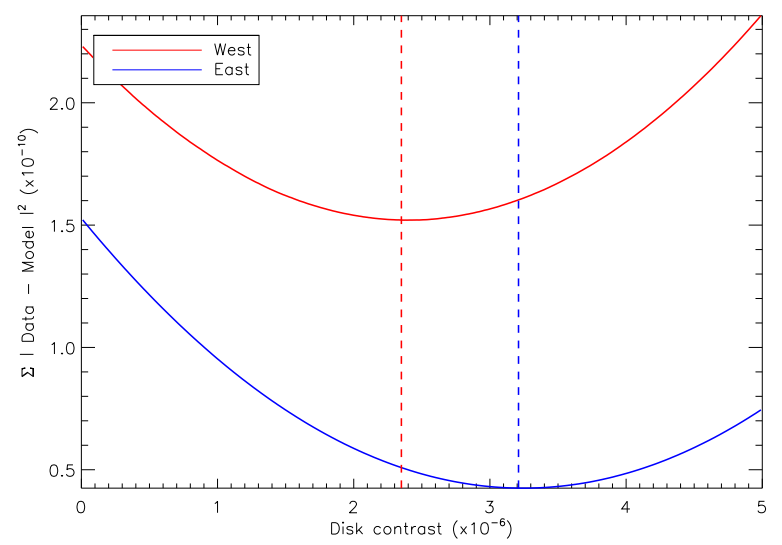

Fig. 8. Optimization criteria versus the surface brightness factor of the model for the south-east (blue) and north-west (red) part of the disc. Dashed lines correspond to the abscissa position of the minimum.

Thébault \& Wu 2008). However, because of the very poor S/N beyond the main ring, the $\alpha_{\text {out }}$ value is only constrained over a narrow radial domain beyond $R_{0}$, for which departures from a -1.5 slope could in fact be obtained even for an unperturbed system (the asymptotical -1.5 slope being reached further out, see Thebault et al. 2012).

A second explanation for an azimuthal over-density is the result of the recent breakup of a massive planetesimal, which releases dusty fragments with highly eccentric orbits all passing through the initial breakup location. This can create an observable long-lived two-sided asymmetry (Jackson et al. 2014; Kral et al. 2015). However, the fainter side (opposite to the breakup location) should in this case be more radially extended than the bright side, whereas we observe the opposite, even though this trend should be taken with caution because the luminosity profile beyond the main ring is poorly constrained in the present images.

An alternative scenario is that the south-west to north-east asymmetry is not due to azimuthal inhomogeneities but to the fact that the disc is eccentric, with its periastron located somewhere in the western side, scattering more stellar light because it is closer to the star. This "pericenter glow" effect has been identified by Wyatt et al. (1999) and is suspected to be witnessed in several discs. More generally, an eccentric ring of parent bodies, coupled to the size-dependent effect of radiation pressure, can create strong brightness asymmetries. The thorough exploration by Lee \& Chiang (2016) showed that, for some disc geometries and viewing angles, asymmetries resembling the one observed here can be obtained (see for example Fig. 9 of that paper). Of course, the eccentric ring scenario also requires an additional force (perturbations by a planetary object or a companion star) to explain this eccentricity. However, as with the giant-breakup scenario, the fainter side of the disc should be radially more extended than the bright side, in apparent contradiction with the present image.

At the present time, the large uncertainties caused by the low $\mathrm{S} / \mathrm{N}$ of our observations do not allow us to easily discriminate between these different scenarios. Future and deeper observations, in particular to constrain the surface brightness beyond the main ring, are clearly needed.

We also report the apparent discrepancy between the disc's radial location inferred here, $\sim 86 \mathrm{au}$, and the one derived by Moór et al. (2016), $58 \pm 13 \mathrm{au}$, by fitting the system's SED. However, this difference can be attributed to the fact that the SED fitting was done assuming black-body temperatures for the grains. As rightfully underlined by Moór et al. (2016) themselves, smaller grains, which are the ones likely to dominate the thermal emission up to mid-IR wavelengths, are ineffective emitters that can be much hotter than black bodies at the same radial distance. As such, the 58 au value should be regarded as a lower limit that could easily be increased by a factor of two with more realistic modelling (Moór et al. 2016), making it compatible with our present measurement. In order to correct this effect, we used the correction factor described in Pawellek \& Krivov (2015) (Eqs. (8) and (9)), and we corrected the dust radius and obtained several values, depending on the dust composition (we need more measurements on the SED to discriminate the dust composition). We found a dust radius of $203.3 \pm 74.0 \mathrm{au}(50 \%$ astrosilicate, $50 \%$ vaccum), $195.7 \pm 65.8$ au (50\% astrosilicate, $50 \%$ ice), $274.2 \pm 110.5$ au (100\% astrosilicate), $226,7 \pm 84.7$ au (50\% astrosilicate, $50 \%$ carbon), and $228.8 \pm 82.7$ au (100\% carbon). However, the radii found with the correction of the SED are more extended than the radius measured with SPHERE. This difference could be explained by the lack of measurements in the SED or because the disc is more extended than what we observe with SPHERE, but unseen or suppressed by the ADI algorithm (which acts as a low spatial frequency filter). Our assumptions for these computations are: the bolometric luminosity of the star $L / L_{\odot}=1.52$ (Pawellek \& Krivov 2015), the dust temperature $T_{\text {dust }}=43 \pm 5$ (Moór et al. 2016), and the coefficients A and B of the $\Gamma$ ratio from Pawellek \& Krivov (2015, Table 4).

\section{Conclusion}

The debris disc around the F9V star HD 160305 was detected and resolved for the first time in scattered light with SPHERE at two different epochs. We also detected several point sources around the central star. In this paper we show that ten of these point sources are background stars, while the last five, only detected in the second epoch, can probably be classified as contaminant background stars as well.

We are able to derive important constraints on the disc's morphology. It has a ring-like structure, and is very inclined $\left(\sim 82^{\circ}\right)$ with respect to the line of sight, with a radius of approximately 86-90 au and an estimated deprojected width of 27 au. We also determine that the southern part of the disc is probably the front side of the ring, appearing much brighter than the northern part due to the forward scattering effect of the dust.

We also detected a two-sided surface brightness asymmetry, between the south-east and the north-west parts. The surface brightness ratio between the two parts is $0.73 \pm 0.18$ and the fractional luminosity of the disc relative to the star is around $1.4 \times 10^{-4}$. Several scenarios could explain this asymmetry, such as the interaction with a massive companion, a recent and massive collision of planetesimals, or a pericenter glow effect due to a potential eccentricity of the disc. However, due to the faintness of the disc in our current data we cannot discriminate between these different scenarios without follow-up observations.

Acknowledgements. SPHERE is an instrument designed and built by a consortium consisting of IPAG (Grenoble, France), MPIA (Heidelberg, Germany), LAM (Marseille, France), LESIA (Paris, France), Laboratoire Lagrange (Nice, France), INAF-Osservatorio di Padova (Italy), Observatoire de Genève (Switzerland), ETH Zurich (Switzerland), NOVA (Netherlands), ONERA (France) and ASTRON (Netherlands) in collaboration with ESO. SPHERE was funded by ESO, with additional contributions from CNRS (France), MPIA (Germany), INAF (Italy), FINES (Switzerland), and NOVA (Netherlands). SPHERE also received funding from the European Commission Sixth and Seventh Framework Programmes as part of the Optical Infrared Coordination Network for Astronomy (OPTICON) under grant number RII3-Ct-2004-001566 for FP6 (2004-2008), grant number 226604 for FP7 (2009-2012), and grant 
number 312430 for FP7 (2013-2016). We also acknowledge financial support from the Programme National de Planétologie (PNP) and the Programme National de Physique Stellaire (PNPS) of CNRS-INSU in France. This work has also been supported by a grant from the French Labex OSUG@2020 (Investissements d'avenir - ANR10 LABX56). The project is supported by CNRS, by the Agence Nationale de la Recherche (ANR-14-CE33-0018). It has also been carried out within the frame of the National Centre for Competence in Research PlanetS supported by the Swiss National Science Foundation (SNSF). M.R.M., H.M.S., and S.D. are pleased to acknowledge this financial support of the SNSF. Finally, this work has made use of the SPHERE Data Centre, jointly operated by OSUG/IPAG (Grenoble), PYTHEAS/LAM/CESAM (Marseille), OCA/Lagrange (Nice) and Observtoire de Paris/LESIA (Paris). We thank P Delorme and E. Lagadec (SPHERE Data Centre) for their efficient help during the data reduction process. R.G. and S.D. acknowledge support from the "Progetti Premiali" funding scheme of the Italian Ministry of Education, University, and Research. This work has been supported by the project PRIN INAF 2016 The Cradle of Life - GENESIS-SKA (General Conditions in Early Planetary Systems for the rise of life with SKA). J.O. and C.P. acknowledge financial support from the ICM (Iniciativa Científica Milenio) via the Núcleo Milenio de Formación Planetaria grant, from the Universidad de Valparaíso. J.O. also acknowledge financial support from Fondecyt (grant 1180395). C.P. also acknowledge financial support from Fondecyt (grant 3190691). F.Me. acknowledges funding from ANR of France under contract number ANR-16-CE31-0013. D.M. acknowledges support from the ESO-Government of Chile Joint Comittee program "Direct imaging and characterization of exoplanets". A.-L.M. acknowledges financial support from the F.R.S.-FNRS.

\section{References}

Augereau, J. C., Lagrange, A. M., Mouillet, D., Papaloizou, J. C. B., \& Grorod, P. A. 1999, A\&A, 348, 557

Augereau, J. C., Nelson, R. P., Lagrange, A. M., Papaloizou, J. C. B., \& Mouillet, D. 2001, A\&A, 370, 447

Baraffe, I., Homeier, D., Allard, F., \& Chabrier, G. 2015, A\&A, 577, A42

Bell, C. P. M., Mamajek, E. E., \& Naylor, T. 2015, MNRAS, 454, 593

Beuzit, J.-L., Vigan, A., Mouillet, D., et al. 2019, A\&A, submitted [arXiv: 1902.04080$]$

Boccaletti, A., Thalmann, C., Lagrange, A.-M., et al. 2015, Nature, 526, 230

Boccaletti, A., Sezestre, E., Lagrange, A.-M., et al. 2018, A\&A, 614, A52

Bonnefoy, M., Perraut, K., Lagrange, A.-M., et al. 2018, A\&A, 618, A63

Chauvin, G., Desidera, S., Lagrange, A.-M., et al. 2017, in SF2A-2017: Proceedings of the Annual meeting of the French Society of Astronomy and Astrophysics, eds. C. Reylé, P. Di Matteo, F. Herpin, et al., 331

Choquet, É., Perrin, M. D., Chen, C. H., et al. 2016, ApJ, 817, L2

Claudi, R. U., Turatto, M., Gratton, R. G., et al. 2008, in Ground-based and Airborne Instrumentation for Astronomy II, Proc. SPIE, 7014, 70143E

Delorme, P., Meunier, N., Albert, D., et al. 2017, in SF2A-2017: Proceedings of the Annual meeting of the French Society of Astronomy and Astrophysics, eds. C. Reylé, P. Di Matteo, F. Herpin, et al., 347

Dohlen, K., Langlois, M., Saisse, M., et al. 2008, in Ground-based and Airborne Instrumentation for Astronomy II, Proc. SPIE, 7014, 70143L

Feldt, M., Olofsson, J., Boccaletti, A., et al. 2017, A\&A, 601, A7

Gagné, J., Mamajek, E. E., Malo, L., et al. 2018, ApJ, 856, 23

Gaia Collaboration (Brown, A. G. A., et al.) 2018, A\&A, 616, A1

Galicher, R., Boccaletti, A., Mesa, D., et al. 2018, A\&A, 615, A92

Guerri, G., Daban, J.-B., Robbe-Dubois, S., et al. 2011, Exp. Astron., 30, 59

Hedman, M. M., \& Stark, C. C. 2015, ApJ, 811, 67

Henyey, L. G., \& Greenstein, J. L. 1941, ApJ, 93, 70

Hernández, J., Hartmann, L., Megeath, T., et al. 2007, ApJ, 662, 1067

Hines, D. C., Schneider, G., Hollenbach, D., et al. 2007, ApJ, 671, L165

Hughes, A. M., Duchêne, G., \& Matthews, B. C. 2018, ARA\&A, 56, 541

Jackson, A. P., Wyatt, M. C., Bonsor, A., \& Veras, D. 2014, MNRAS, 440, 3757

Kalas, P., Graham, J. R., \& Clampin, M. 2005, Nature, 435, 1067

Kalas, P., Fitzgerald, M. P., \& Graham, J. R. 2007, ApJ, 661, L85
Kalas, P., Graham, J. R., Chiang, E., et al. 2008, Science, 322, 1345

Kalas, P. G., Rajan, A., Wang, J. J., et al. 2015, ApJ, 814, 32

Kiraga, M. 2012, Acta Astron., 62, 67

Kiss, L. L., Moór, A., Szalai, T., et al. 2011, MNRAS, 411, 117

Kral, Q., Thébault, P., Augereau, J.-C., Boccaletti, A., \& Charnoz, S. 2015, A\&A, 573, A39

Kral, Q., Marino, S., Wyatt, M. C., Kama, M., \& Matra, L. 2019, MNRAS, accepted [arXiv:1811.08439]

Kuchner, M. J., \& Holman, M. J. 2003, ApJ, 588, 1110

Lagrange, A.-M., Bonnefoy, M., Chauvin, G., et al. 2010, Science, 329, 57

Lagrange, A.-M., Langlois, M., Gratton, R., et al. 2016, A\&A, 586, L8

Langlois, M., Vigan, A., Moutou, C., et al. 2013, in Proceedings of the Third AO4ELT Conference, eds. S. Esposito, \& L. Fini, 63

Langlois, M., Vigan, A., Dohlen, K., et al. 2014, in Ground-based and Airborne Instrumentation for Astronomy V, Proc. SPIE, 9147, 91479P

Lee, E. J., \& Chiang, E. 2016, ApJ, 827, 125

Lee, J., \& Song, I. 2018, MNRAS, 475, 2955

Lyra, W., \& Kuchner, M. 2013, Nature, 499, 184

Macintosh, B., Graham, J. R., Ingraham, P., et al. 2014, Proc. Natl. Acad. Sci., 111, 12661

Maire, A.-L., Langlois, M., Dohlen, K., et al. 2016, in Ground-based and Airborne Instrumentation for Astronomy VI, Proc. SPIE, 9908, 990834

Malo, L., Doyon, R., Lafrenière, D., et al. 2013, ApJ, 762, 88

Mamajek, E. E., \& Bell, C. P. M. 2014, MNRAS, 445, 2169

Marois, C., Lafrenière, D., Doyon, R., Macintosh, B., \& Nadeau, D. 2006, ApJ, 641, 556

Marois, C., Correia, C., Galicher, R., et al. 2014, in Adaptive Optics Systems IV, Proc. SPIE, 9148, 91480U

Mesa, D., Gratton, R., Zurlo, A., et al. 2015, A\&A, 576, A121

Messina, S., Millward, M., Buccino, A., et al. 2017, A\&A, 600, A83

Milli, J., Mouillet, D., Lagrange, A.-M., et al. 2012, A\&A, 545, A111

Milli, J., Vigan, A., Mouillet, D., et al. 2017, A\&A, 599, A108

Moór, A., Henning, T., Juhász, A., et al. 2015, ApJ, 814, 42

Moór, A., Kóspál, Á., Ábrahám, P., et al. 2016, ApJ, 826, 123

Mouillet, D., Larwood, J. D., Papaloizou, J. C. B., \& Lagrange, A. M. 1997, MNRAS, 292, 896

Neuhäuser, R., Hohle, M. M., Ginski, C., et al. 2015, MNRAS, 448, 376

Olofsson, J., Samland, M., Avenhaus, H., et al. 2016, A\&A, 591, A108

Pavlov, A., Feldt, M., \& Henning, T. 2008, in Astronomical Data Analysis Software and Systems XVII, eds. R. W. Argyle, P. S. Bunclark, \& J. R. Lewis, ASP Conf. Ser., 394, 581

Pawellek, N., \& Krivov, A. V. 2015, MNRAS, 454, 3207

Pecaut, M. J., \& Mamajek, E. E. 2013, ApJS, 208, 9

Pecaut, M. J., \& Mamajek, E. E. 2016, MNRAS, 461, 794

Perrot, C., Boccaletti, A., Pantin, E., et al. 2016, A\&A, 590, L7

Poppenhaeger, K., Auchettl, K., \& Wolk, S. J. 2017, MNRAS, 468, 4018

Reche, R., Beust, H., Augereau, J.-C., \& Absil, O. 2008, A\&A, 480, 551

Richert, A. J. W., Getman, K. V., Feigelson, E. D., et al. 2018, MNRAS, 477, 5191

Robin, A. C., Reylé, C., Derrière, S., \& Picaud, S. 2003, A\&A, 409, 523

Schneider, A. 2013, Ph.D. Thesis, University of Georgia, USA

Schneider, G., Smith, B. A., Becklin, E. E., et al. 1999, ApJ, 513, L127

Sissa, E., Olofsson, J., Vigan, A., et al. 2018, A\&A, 613, L6

Smith, W. H. 1987, PASP, 99, 1344

Song, I., Zuckerman, B., \& Bessell, M. S. 2012, AJ, 144, 8

Soummer, R., Pueyo, L., \& Larkin, J. 2012, ApJ, 755, L28

Strubbe, L. E., \& Chiang, E. I. 2006, ApJ, 648, 652

Thébault, P. 2009, A\&A, 505, 1269

Thébault, P., \& Wu, Y. 2008, A\&A, 481, 713

Thebault, P., Kral, Q., \& Ertel, S. 2012, A\&A, 547, A92

van Leeuwen, F. 2007, A\&A, 474, 653

Vigan, A., Moutou, C., Langlois, M., et al. 2010, in Ground-based and Airborne Instrumentation for Astronomy III, Proc. SPIE, 7735, 77352X

Williams, J. P., \& Cieza, L. A. 2011, ARA\&A, 49, 67

Wyatt, M. C., Dermott, S. F., Telesco, C. M., et al. 1999, ApJ, 527, 918 


\section{Appendix A: Derivation of the stellar rotation axis}

To derive the inclination of the stellar rotation axis, $i_{\star}$, we used the formula

$\sin \left(i_{\star}\right)=\frac{P \times v_{\sin i}}{50.576 \times R}$,

where $P$ is the period of the stellar rotation (in days), $v_{\sin i}$ the projected rotational velocity (in $\mathrm{km} \mathrm{s}^{-1}$ ), $R$ the stellar radius (in solar radii), and 50.576 a constant to transform units from centimetre and s to solar radii and days. For HD 160305, we assume $P=1.336 \pm 0.008$ days (Messina et al. 2017), $v_{\sin i}=37 \mathrm{~km} \mathrm{~s}^{-1}$ (as no error bars were reported, we assume $10 \%$ of uncertainties in the $v_{\sin i}$ value). We derived a stellar luminosity $L=1.6 \pm 0.2 L_{\odot}$ and a stellar radius $R=1.15 \pm 0.18 R_{\odot}$ using the new Gaia distance from the DR2 $(65.51 \pm 0.23 \mathrm{pc}$, Gaia Collaboration 2018), the magnitude $V=8.405$ (Kiraga 2012), an effective temperature $T=6065 \mathrm{~K}$ (Pecaut \& Mamajek 2013), and a bolometric correction $\mathrm{BC}_{V}=-0.05$ magnitude (Pecaut \& Mamajek 2013) . Finally, we can derive a stellar rotation axis of $i_{\star}=58_{-10}^{\circ+18}$. This value is different from the disc inclination found $\left(82^{\circ}\right)$ and could suggest a misalignment between the stellar equatorial plane and the disc inclination. It is possible that the asymmetry observed in the disc was a consequence of this misalignment. However, in this paper we have no proof for this hypothesis, which could be investigated in a future work.

\section{Appendix B: Additional data}

Table B.1. Setup of observations.

\begin{tabular}{ccccccccccc}
\hline \hline $\begin{array}{c}\text { Date } \\
-\end{array}$ & $\begin{array}{c}\text { Mode } \\
-\end{array}$ & $\begin{array}{c}\text { Filter } \\
-\end{array}$ & $\begin{array}{c}\text { DIT } \\
(\mathrm{s})\end{array}$ & $\begin{array}{c}\text { NDIT } \\
-\end{array}$ & $\begin{array}{c}T_{\exp } \\
(\mathrm{s})\end{array}$ & $\begin{array}{c}\text { Rotation } \\
\left(^{\circ}\right)\end{array}$ & $\begin{array}{c}\text { Seeing } \\
\left({ }^{\prime \prime}\right)\end{array}$ & $\begin{array}{c}\tau_{0} \\
(\mathrm{~ms})\end{array}$ & $\begin{array}{c}\text { TN } \\
\left(^{\circ}\right)\end{array}$ & $\begin{array}{c}\text { pixscale } \\
(\mathrm{mas})\end{array}$ \\
\hline $2015-05-13^{(a)}$ & IRDIS & $H 2+H 3$ & 64 & 64 & 4096 & 34.58 & $0.7-1.0$ & $3-4$ & $-1.712 \pm 0.063$ & $12.242 \pm 0.062$ \\
$2015-05-13^{(a)}$ & IFS & $Y J$ & 64 & 64 & 4096 & 34.58 & $0.7-1.0$ & $3-4$ & $-1.712 \pm 0.063$ & $7.46 \pm 0.02$ \\
$2016-05-23^{(b)}$ & IRDIS & $B B_{-} H$ & 32 & 144 & 4608 & 40.20 & $0.5-0.7$ & $3-6$ & $-1.675 \pm 0.080$ & $12.247 \pm 0.017$ \\
$2016-05-23^{(b)}$ & IFS & $Y J$ & 32 & 144 & 4608 & 40.20 & $0.5-0.7$ & $3-6$ & $-1.675 \pm 0.080$ & $7.46 \pm 0.02$ \\
\hline
\end{tabular}

Notes. Column 1 - Date of the observation $\left({ }^{(a)}\right.$ is programme 95.C-0298(A), ${ }^{(b)}$ is programme 97.C-0865). Column 2 - Observation mode (IRDIS or IFS). Column 3 - Filter used. Column 4 - Individual time for each individual image. Column 5 - Total number of images. Column 6 - Total time of the observation. Column 7 - Total field rotation of the observation. Column 8 - Seeing measured by the telescope. Column $9-$ Coherence time measured at the telescope. Column 10 - Value of the true-north orientation. Column 11 - Angular size of a pixel (Maire et al. 2016).

Table B.2. Photometric and astrometric measurements of point sources detected in the first epoch (Cols. 1-5) and the second epoch (Cols. 6-8).

\begin{tabular}{|c|c|c|c|c|c|c|c|c|c|}
\hline $\begin{array}{l}\# \\
-\end{array}$ & $\begin{array}{c}m_{H 2} \\
-\end{array}$ & $\begin{array}{c}m_{H 3} \\
-\end{array}$ & $\begin{array}{l}\text { Separation } \\
\text { (mas) }\end{array}$ & $\begin{array}{l}\text { PA } \\
\left({ }^{\circ}\right)\end{array}$ & $\begin{array}{c}m_{H} \\
-\end{array}$ & $\begin{array}{l}\text { Separation } \\
\left({ }^{\prime \prime}\right)\end{array}$ & $\begin{array}{l}\text { PA } \\
\left({ }^{\circ}\right)\end{array}$ & $\begin{array}{c}\text { Probability } \\
\%\end{array}$ & $\begin{array}{c}\text { Status } \\
-\end{array}$ \\
\hline 1 & $16.77 \pm 0.08$ & $16.78 \pm 0.08$ & $3338.1 \pm 17.0$ & $331.33 \pm 0.07$ & $16.50 \pm 0.15$ & $3392.5 \pm 4.9$ & $332.01 \pm 0.09$ & 13.5 & BS \\
\hline 2 & $20.40 \pm 0.08$ & $20.28 \pm 0.08$ & $3451.6 \pm 17.6$ & $3.43 \pm 0.39$ & $20.11 \pm 0.15$ & $3519.7 \pm 5.1$ & $3.57 \pm 0.28$ & 71.5 & $\mathrm{BS}$ \\
\hline 3 & $22.69 \pm 0.19$ & $22.56 \pm 0.15$ & $3694.1 \pm 20.5$ & $115.71 \pm 0.12$ & $22.29 \pm 0.18$ & $3677.7 \pm 7.7$ & $114.72 \pm 0.10$ & 93.7 & $\mathrm{BS}$ \\
\hline 4 & $19.08 \pm 0.08$ & $19.02 \pm 0.08$ & $4091.7 \pm 20.8$ & $186.52 \pm 0.16$ & $18.79 \pm 0.15$ & $4037.2 \pm 5.8$ & $186.60 \pm 0.15$ & 57.9 & BS \\
\hline 5 & $22.72 \pm 0.16$ & $22.57 \pm 0.21$ & $4143.9 \pm 22.7$ & $327.61 \pm 0.17$ & $22.31 \pm 0.21$ & $4193.3 \pm 7.8$ & $328.20 \pm 0.13$ & 97.4 & $\mathrm{BS}$ \\
\hline 6 & $17.53 \pm 0.08$ & $17.58 \pm 0.08$ & $4154.8 \pm 21.1$ & $58.29 \pm 0.07$ & $17.27 \pm 0.15$ & $4191.2 \pm 5.9$ & $57.66 \pm 0.08$ & 30.9 & $\mathrm{BS}$ \\
\hline 7 & $21.74 \pm 0.10$ & $21.74 \pm 0.10$ & $5046.4 \pm 25.8$ & $150.21 \pm 0.09$ & $21.42 \pm 0.17$ & $4992.9 \pm 7.5$ & $149.86 \pm 0.09$ & 98.1 & BS \\
\hline 8 & $19.93 \pm 0.08$ & $19.93 \pm 0.08$ & $5376.7 \pm 27.3$ & $60.42 \pm 0.07$ & $19.64 \pm 0.15$ & $5415.8 \pm 7.7$ & $59.89 \pm 0.08$ & 90.0 & BS \\
\hline 9 & $21.98 \pm 0.13$ & $21.93 \pm 0.14$ & $5616.8 \pm 28.9$ & $201.19 \pm 0.12$ & $22.27 \pm 0.19$ & $5554.2 \pm 9.0$ & $201.46 \pm 0.12$ & 99.8 & BS \\
\hline 10 & $21.20 \pm 0.19$ & $20.71 \pm 0.10$ & $7009.0 \pm 36.0$ & $110.91 \pm 0.07$ & $20.67 \pm 0.16$ & $6985.8 \pm 10.2$ & $110.40 \pm 0.08$ & 99.6 & BS \\
\hline 11 & - & - & - & - & $23.10 \pm 0.22$ & $6198.7 \pm 15.8$ & $164.53 \pm 0.28$ & 100.0 & $\mathrm{BS}$ \\
\hline 12 & - & - & - & - & $17.49 \pm 0.15$ & $7307.9 \pm 10.2$ & $155.30 \pm 0.08$ & 72.3 & $\mathrm{BS}$ \\
\hline 13 & - & - & - & - & $20.92 \pm 0.23$ & $7436.4 \pm 11.6$ & $154.97 \pm 0.10$ & 100.0 & $\mathrm{BS}$ \\
\hline 14 & - & - & - & - & $19.59 \pm 0.15$ & $7778.4 \pm 11.0$ & $157.70 \pm 0.08$ & 98.8 & $\mathrm{BS}$ \\
\hline 15 & - & - & - & - & $23.60 \pm 0.50$ & $2984.7 \pm 23.2$ & $105.22 \pm 0.30$ & 89.0 & $\mathrm{BS}$ \\
\hline
\end{tabular}

Notes. Column 1 is the label in the images shown in Fig. 1, Cols. 2 and 3 are respectively the $H 2$ and $H 3$ relative magnitudes of point sources, Col. 4 is the angular separation to the star of the point sources for the first epoch, Col. 5 is the position angle of the point sources $\left(0^{\circ}\right.$ is north and it is counted positive from north to east) for the first epoch, Col. 6 is the $B B \_H$ relative magnitude of point sources, Col. 7 is the angular separation to the star of the point sources for the second epoch, Col. 8 is the position angle of the point sources for the second epoch, Col. 9 is the probability of each candidate to be a contaminant background star, based on the Besançon Galaxy Model (Robin et al. 2003), Col. 10 is the current status of candidates (BS: background star). 